\title{
Imagem radiográfica da cavidade torácica de cães Golden Retriever acometidos pela Distrofia Muscular ${ }^{1}$
}

\author{
Flávio R. Alves ${ }^{2 *}$, Matheus L.T. Feitosa², André Gatti², Leandro Fadel ${ }^{2}$, \\ Silvana M. Unruh ${ }^{3}$, Carlos E. Ambrósio ${ }^{3}$, Franklin A. Sterman ${ }^{3}$, Ana C.B.C.F. \\ Pinto $^{3}$ e Maria A. Miglino 3
}

\begin{abstract}
Alves F.R., Feitosa M.L.T., Gatti A., Fadel L., Unruh S.M., Ambrósio C.E., Sterman F.A., Pinto A.C.B.C.F. \& Miglino M.A. 2009. [Radiologic images of the thoracic cavity of Golden Retriever dogs affected by muscular dystrophy.] Imagem radiográfica da cavidade torácica de cães Golden Retriever acometidos pela Distrofia Muscular. Pesquisa Veterinária Brasileira 29(2):99-104. Departamento de Cirurgia, Faculdade de Medicina Veterinária e Zootecnia, Universidade de São Paulo, Av. Prof. Dr. Orlando Marques de Paiva 87, São Paulo, SP 05508-270, Brazil. E-mail: flaviovet@usp.br

Duchenne Muscular Dystrophy (DMD) is a genetic disorder with clinical signs of muscular weaknesses and progressive atrophy. Golden Retriever dogs show similar genotypic and phenotypic characteristics to Human Muscular Dystrophy and are considered a proper animal model for DMD studies. Latero-lateral and dorso-ventral thoracic radiographies were obtained from 10 Golden Retriever dogs affected by muscular dystrophy, to investigate possible radiographic alterations. Thorax radiographic examination revealed (a) interstitial and alveolar pattern, (b) initial phases of pneumonia and pulmonary edema, (c) cardiomegaly as a principal alteration in the thoracic cavity, (d) megaesophagus displacing the trachea and heart silhouette, and (e) cranial protrusion of the diaphragm lining into the thorax with development of a hiatus hernia displacing the stomach to the caudal mediastinum. Postmortem examination showed pleural effusion, pulmonary emphysema, degenerative and metaplasic processes in the diaphragm and intercostal muscles. Radiographic examination was considered essential for the diagnosis of cardiac and respiratory disease in Golden Retriever dogs affected by muscular dystrophy, and to identify the primary pulmonary process and to provide the establishment of suitable therapeutic treatment, with a reserved prognosis in advanced stage of the disease.
\end{abstract}

INDEX TERMS: Muscular dystrophy, Duchenne, Golden Retriever, dog, radiology.

RESUMO.- A distrofia muscular de Duchenne (DMD) é uma doença de origem genética, cuja principal manifestação clínica é enfraquecimento e atrofia progressiva dos músculos. Os cães da raça Golden Retriever podem apresentar distrofia muscular, com características genotípicas e fenotípicas muito próximas à distrofia muscular humana, sendo considerado o modelo animal mais apropriado

\footnotetext{
${ }^{1}$ Recebido em 3 de novembro de 2007.

Aceito para publicação em 5 de setembro de 2008.

2 Programa de Pós-Graduação em Anatomia dos Animais Domésticos e Silvestres, Departamento de Cirurgia, Faculdade de Medicina Veterinária e Zootecnia (FMVZ), Universidade de São Paulo (USP), Av. Prof. Dr. Orlando Marques de Paiva 87, Cidade Universitária, São Paulo, SP 05508-270, Brasil. *Autor para correspondência: flaviovet@usp.br

${ }^{3}$ Departamento de Cirurgia, FMVZ, USP, São Paulo, SP.
}

para o estudo da DMD. Foram realizadas radiografias torácicas látero-laterais e dorsoventrais de 10 cães Golden Retriever afetados pela distrofia muscular, com o objetivo de relatar as alterações radiográficas associadas a essa patologia. O exame radiográfico da cavidade torácica evidenciou: (a) padrão pulmonar intersticial e alveolar predominante, (b) um quadro de pneumonia e edema pulmonar em fase inicial, (c) a cardiomegalia como o principal achado de comprometimento circulatório na cavidade torácica, (d) O megaesôfago torácico foi observado deslocando a traquéia e silhueta cardíaca ventralmente e, (e) a cúpula diafragmática apresentou modificação morfológica, mostrando protrusão para o interior da cavidade torácica e hérnia hiatal, com deslocamento do estômago para o espaço mediastino caudal. Os achados de necropsia 
evidenciaram efusão pleural e enfisema pulmonar e lesões compatíveis com processos degenerativos e metaplásicos da musculatura diafragmática e intercostal. A avaliação radiográfica constituiu-se como um meio diagnóstico auxiliar essencial na identificação de doença cardíaca e respiratória em cães Golden Retriever acometidos pela Distrofia Muscular, capaz de identificar processos pneumônicos primários, permitindo o estabelecimento de terapêutica adequada de tratamento, com prognóstico reservado nos estágios mais avançados desta alteração.

TERMOS DE INDEXAÇÃO: Distrofia muscular, Duchenne, cão, Golden Retriever, radiologia.

\section{INTRODUÇÃO}

A distrofia muscular foi descrita em humanos como uma doença de origem genética, cuja característica principal é o enfraquecimento e atrofia progressiva dos músculos, prejudicando os movimentos e levando o portador à cadeira de rodas. Ela é uma doença motora que se diferencia das demais porque qualquer esforço muscular que leve ao mínimo de fadiga contribui para a deterioração do tecido muscular. Isto acontece porque o defeito genético ocorre pela ausência ou formação inadequada de proteínas essenciais para o funcionamento da fisiologia da célula muscular (Cooper et al. 1988, Valentine et al.1992).

De acordo com Bergman et al. (2002) e Nguyen et al. (2002), no humano a Distrofia Muscular de Duchenne pode ser caracterizada em três fases distintas (inicial, intermediária e tardia). As complicações respiratórias iniciam-se com o desenvolvimento da fase tardia, a partir do agravamento das deformidades de coluna, que chega a rodar até em um ângulo de até $70^{\circ}$. Como conseqüência, um pulmão fica mais hiperventilado do que o outro. $\mathrm{O}$ acúmulo de secreção leva a perda de reflexo do bocejo e tosse, dificultando a higiene brônquica (Strober 2006).

Em crianças a insuficiência respiratória tornou-se a maior causa de mortalidade. A paresia ou paralisia dos músculos auxiliares da respiração, particularmente dos músculos da parede abdominal e dos músculos grande dorsal, impossibilitando a expiração e inspiração, e conseqüentemente a eliminação de muco pelas vias aéreas. $\mathrm{Na}$ fase inicial da doença o diafragma e a musculatura facial são as únicas estruturas capazes de realizar uma contração ativa (Ronnblon \& Danielsson 2004).

As infecções pulmonares bacterianas ou virais podem causar enfermidade mais severa, como a pneumonia. A diminuição da atividade e esforço debilitante para tossir propicia um acúmulo de secreção, causando assim obstrução das vias aéreas. Os processos obstrutivos, a longo prazo, levam a colabamentos de lobos pulmonares (atelectasia). A hipoventilação crônica tem sido descoberta em pacientes com distrofia muscular. (Bergman et al. 2002, Nguyen et al. 2002).

O cão Golden Retriever portador da distrofia muscular é considerado o modelo animal mais apropriado para o estudo da Distrofia muscular Humana (DMD), devido a sua analogia, tanto em relação ao genótipo quanto ao fenótipo (Cooper et al. 1988, Valente et al. 1992). Nestes animais o diagnóstico da distrofia pode ser realizado com um dia de vida, através da elevação da creatina cinase (CK) ou pela genotipagem por reação em cadeia de polimerase. Quando músculos são submetidos à avaliação por imunohistoquímica, nota-se a ausência da distrofina e proteínas associadas (Bergman et al. 2002, Nguyen et al. 2002).

O perfil da doença, no homem e no cão, se caracteriza pelo ciclo de degeneração e regeneração das fibras musculares, até que se esgote a capacidade regenerativa e que a fibrose progressiva encontre-se estabelecida (Hulland 2003).

Os sinais clínicos podem ser observados no cão Golden Retriever a partir do nascimento e as alterações evoluem rapidamente entre os três e seis meses de idade. Observam-se, com maior freqüência, a disfagia, enrijecimento dos membros pélvicos, com atrofia e hipertrofia muscular seletiva, desvio do eixo de membros, hipertrofia da língua, fraqueza muscular e contraturas (Kornegay et al. 1988). As alterações cardíacas do miocárdio são observadas principalmente em cães afetados adultos (Valentine et al. 1986).

Myazato (2005) demonstrou que a mortalidade foi alta nos animais jovens, ocorrendo entre os sete e nove meses de idade. Nesse estudo, oito animais tiveram causa mortis por falência respiratória decorrente da distrofia muscular. Quando uma doença respiratória aguda encontra-se instalada, o neonato morre durante as duas primeiras semanas de vida (Nguyen et al 2002, Valentine et al. 1991).

Em um estudo de 11 cães afetados pela doença revelou que $91 \%$ destes apresentaram aumento de duas a quatro vezes na espessura do diafragma, ocorrendo nas suas inserções. A região central apresentava-se atrofiada, permitindo a formação da hérnia diafragmática em três animais e ruptura em dois deles (Myazato 2005)

Em um estudo morfológico de cães GRMD, aos seis anos de idade, entre as diversas amostras de músculo esquelético estudados, o diafragma apresentou o mais severo aumento de fibras necróticas com presença de extensa fibrose endomisial e perimisial, ocorrendo infiltrado de tecido adiposo (Valentine et al. 1990).

Em cães distróficos o músculo esofágico encontra-se regularmente afetado. Podem ser observadas fibras musculares degeneradas de tamanho variado e fibrose, evoluindo para megaesôfago cervical, torácico ou ambos, na dependência da evolução do problema (Valentine et al. 1990, Hulland 2003).

A contribuição das doenças cardiorespiratórias para a complicação do quadro terminal da doença na maioria das vezes leva a morte desses animais. Destaca-se então a necessidade de difusão do conhecimento de métodos diagnósticos auxiliares como o exame radiográfico da cavidade torácica que apontem os primeiros sinais de doença cardíaca, respiratória ou ambas, permitindo a adoção de medidas terapêuticas eficazes para a resolução e manutenção da qualidade de vida desses animais. 


\section{MATERIAL E MÉTODOS}

Foram realizados exames radiográficos em 10 cães Golden Retriever machos, com idades variando entre 6 meses e 5 anos de idade, acometidos pela Distrofia Muscular, integrantes da colônia Golden Retriever Muscular Distrophy (GRMD, Brazil), localizada no Setor de Anatomia dos Animais Domésticos e Silvestres da Faculdade de Medicina Veterinária e Zootecnia da Universidade de São Paulo (FMVZ-USP). As radiografias do tórax e abdome foram realizadas utilizando-se projeções látero-laterais direita e esquerda e radiografias dorsoventrais, utilizando-se um aparelho de raios-x Ray Tec de $300 \mathrm{~mA}$, situado nas dependências do Hospital Veterinário da FMVZ-USP. Para os exames foram utilizados fatores de exposição da ordem de $55 \mathrm{kVp}$ e 2,5mAs para as radiografias laterais, aumentando-se $5 \mathrm{kVps}$ para as radiografias dorsoventrais. Os filmes foram revelados em processadora automática Macrotec $M x-2$. Os resultados foram documentados para posterior análise dos achados radiográficos. Todos os cães foram submetidos à avaliação clínica diária, sendo observados desde o momento do nascimento, sendo realizados exames laboratoriais quando julgado necessário.

\section{RESULTADOS}

Os parâmetros clínicos dos animais utilizados no estudo foram avaliados, revelando níveis de desidratação moderados, com parâmetros fisiológicos mantidos dentro dos seus níveis normais. Apenas dois animais apresentaram à auscultação áreas de silêncio pulmonar ou estertores variantes (seco a úmido), na dependência do acumulo de líquido nas vias respiratórias. Seus quadros seguiram associados à febre $\left(39,5-39,8^{\circ} \mathrm{C}\right)$, inapetência e prostração, demonstrando sinais compatíveis com pneumonia, posteriormente confirmados através do exame radiográfico. Foi procedida antibioticoterapia para o tratamento do processo pneumônico nestes animais enfermos, os quais evoluíram para a morte 07 dias após o início dos sintomas.

O exame radiográfico da cavidade torácica de cães Golden Retriever acometidos pela Distrofia muscular revelou predominância de padrão intersticial evoluindo para um padrão alveolar em lobos pulmonares caudais, com-

Fig.1. (A) Radiografia látero-lateral do tórax, onde se observam campos pulmonares com padrão misto (intersticial e alveolar), compatível com edema pulmonar (setas brancas). Chama atenção a densidade radiográfica presente cranial à cúpula diafragmática, sugestiva de hérnia de hiato (contorno em amarela). (B) Broncograma aéreos observados em lobo pulmonar cranial direito (setas brancas), em lobo cranial esquerdo, a presença de broncograma aéreo associado a infiltrado peri-bronquial (setas amarelas). Em (C) observa-se consolidação pulmonar esquerda de padrão alveolar intenso (pneumonia) (setas azuis).
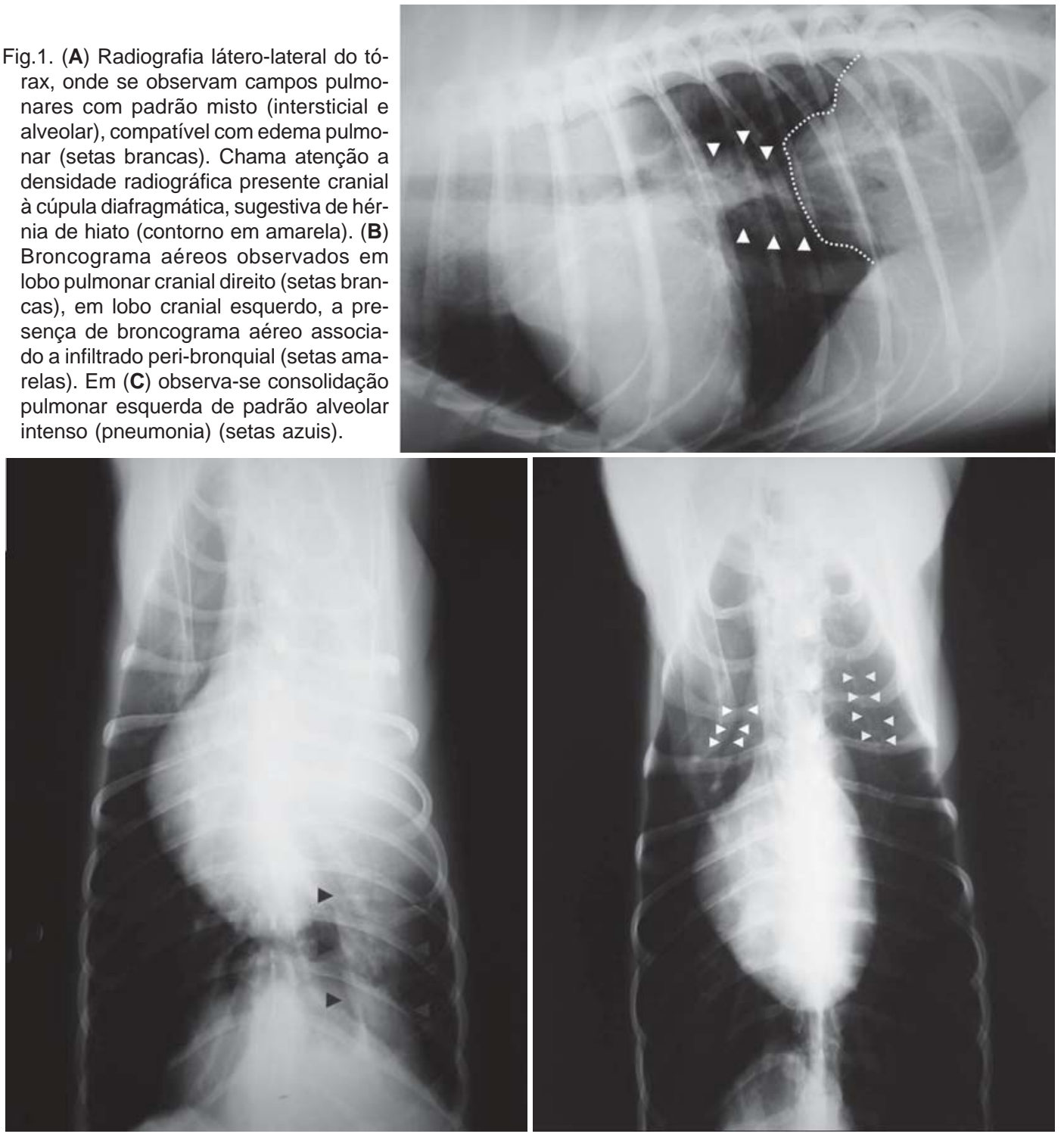
Fig.2. (A) Radiografia látero-lateral do tórax demonstrando a posição verticalizada (ângulo de $90^{\circ}$ ) do coração devido ao aumento cardíaco de átrio e ventrículo esquerdo (contorno em amarela). (B) Observa-se a posição do coração em formato de "D" invertido, caracterizando cardiomegalia.
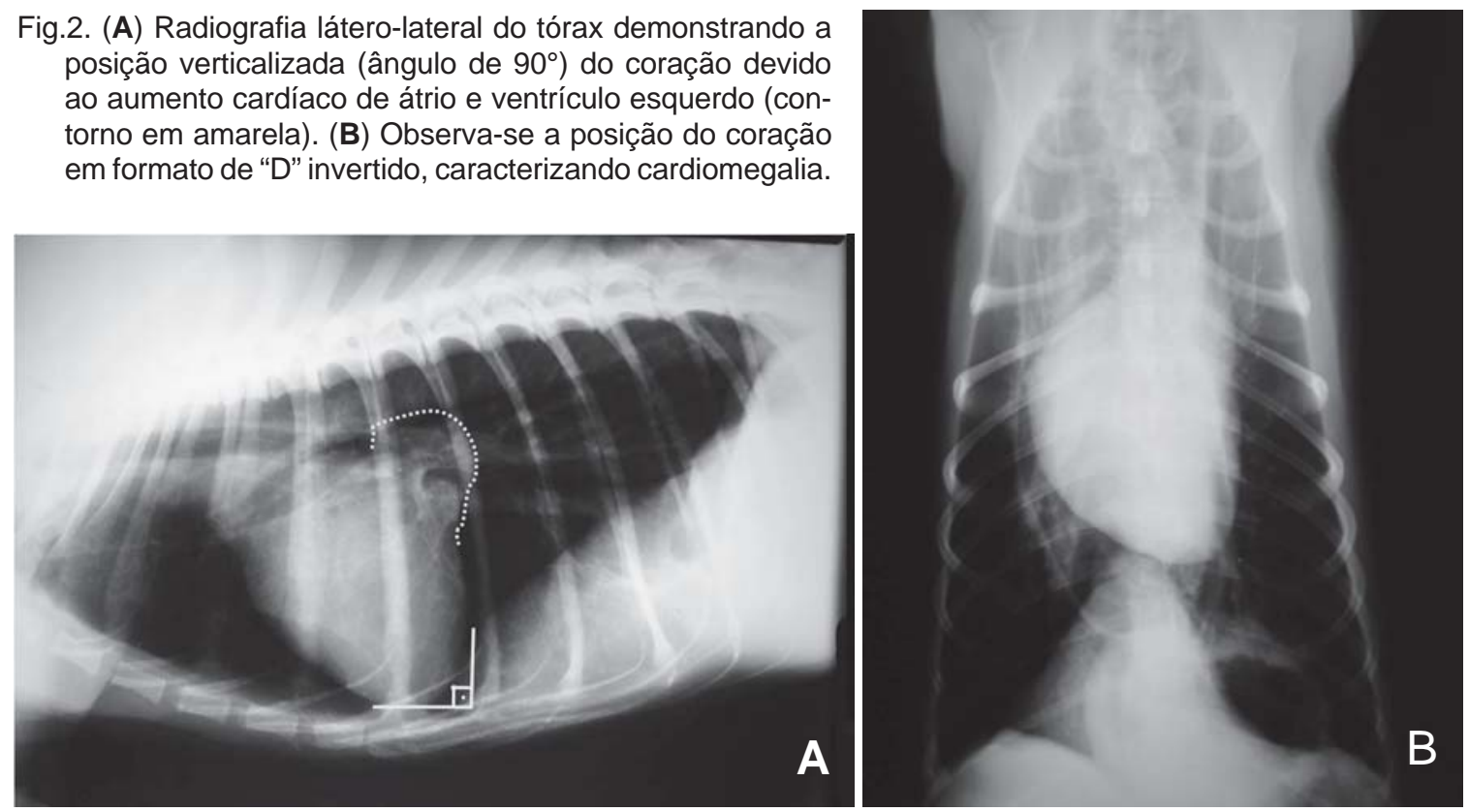

patíveis com edema pulmonar (Fig.1a). Inúmeros broncogramas aéreos foram observadas nos dois quadros mais graves, associados a áreas de consolidação pulmonar (Fig.1b). Em todos os animais foi observada densidade radiográfica presente cranial à cúpula diafragmática, su- gestiva de hérnia hiatal (Fig.1a). Nos animais que evoluíram para quadros mais graves, observou-se a presença de uma intensa área de consolidação observada em toda a extensão do campo pulmonar esquerdo, em imagem compatível com quadro de pneumonia (Fig.1c).
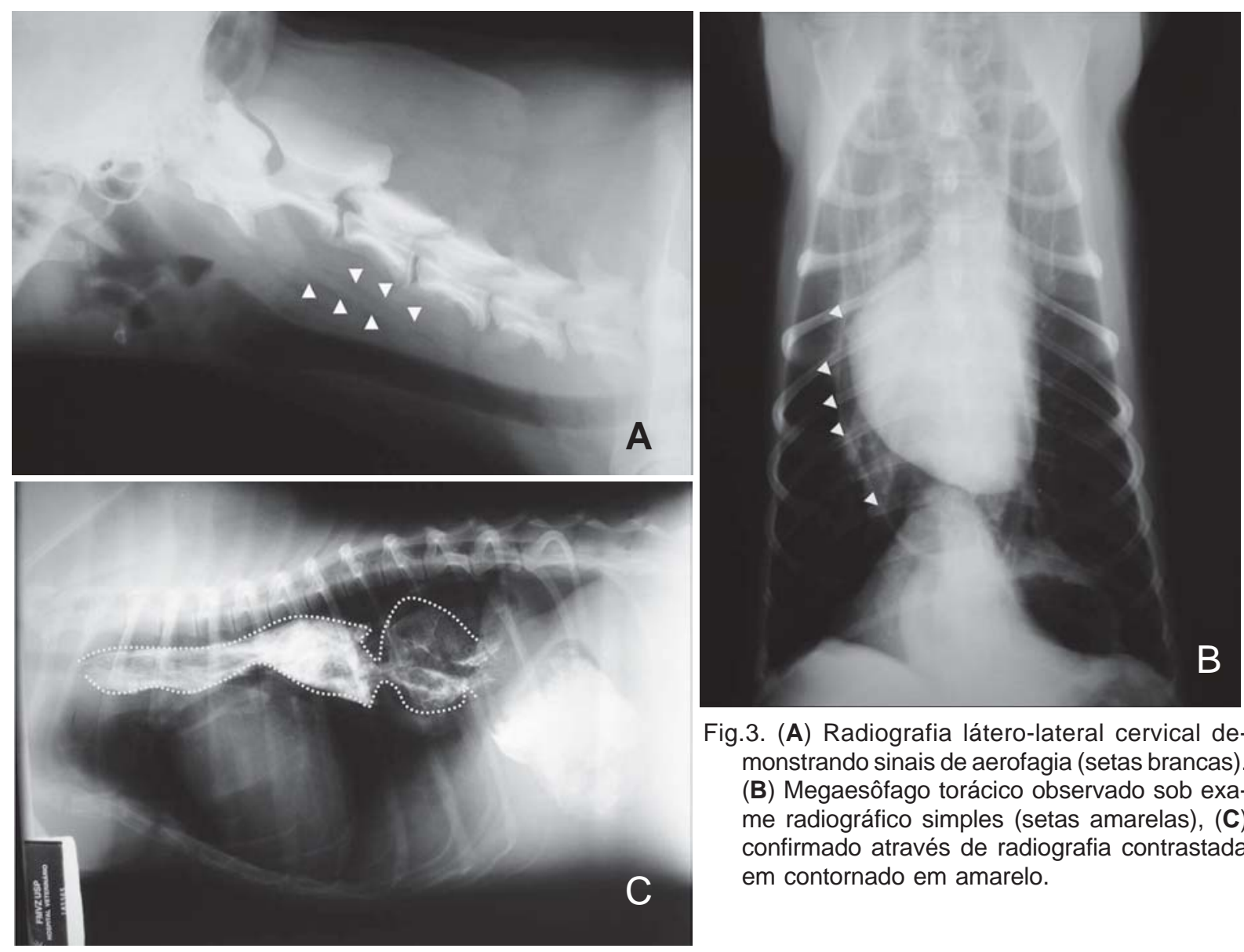

Fig.3. (A) Radiografia látero-lateral cervical demonstrando sinais de aerofagia (setas brancas). (B) Megaesôfago torácico observado sob exame radiográfico simples (setas amarelas), (C) confirmado através de radiografia contrastada em contornado em amarelo. 


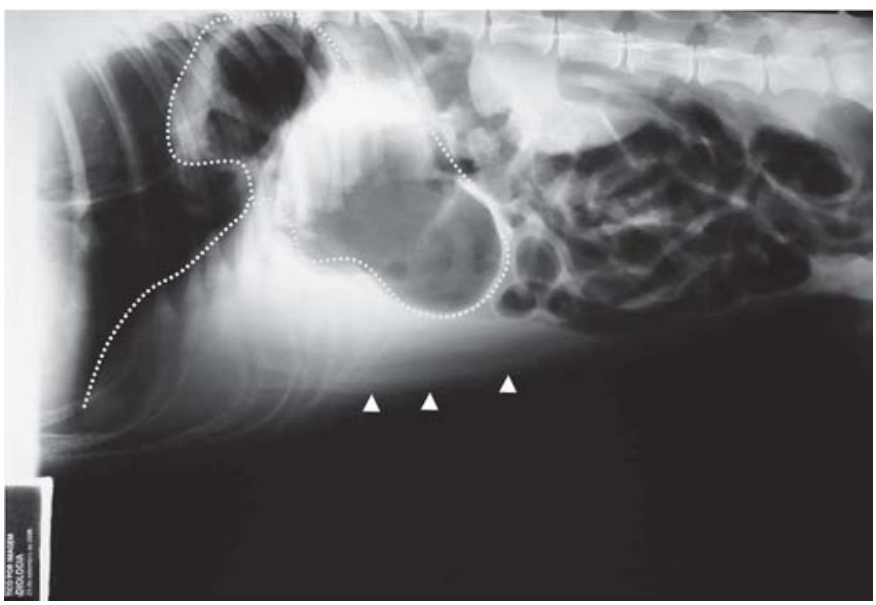

Fig.4. Radiografia látero-lateral do tórax demonstrando a protrusão do estômago (contornado em amarelo) através da cúpula diafragmática (contornado em branco). Nota-se ainda hepatomegalia evidente, com os bordos hepáticos ultrapassando grande distância do gradil costal.
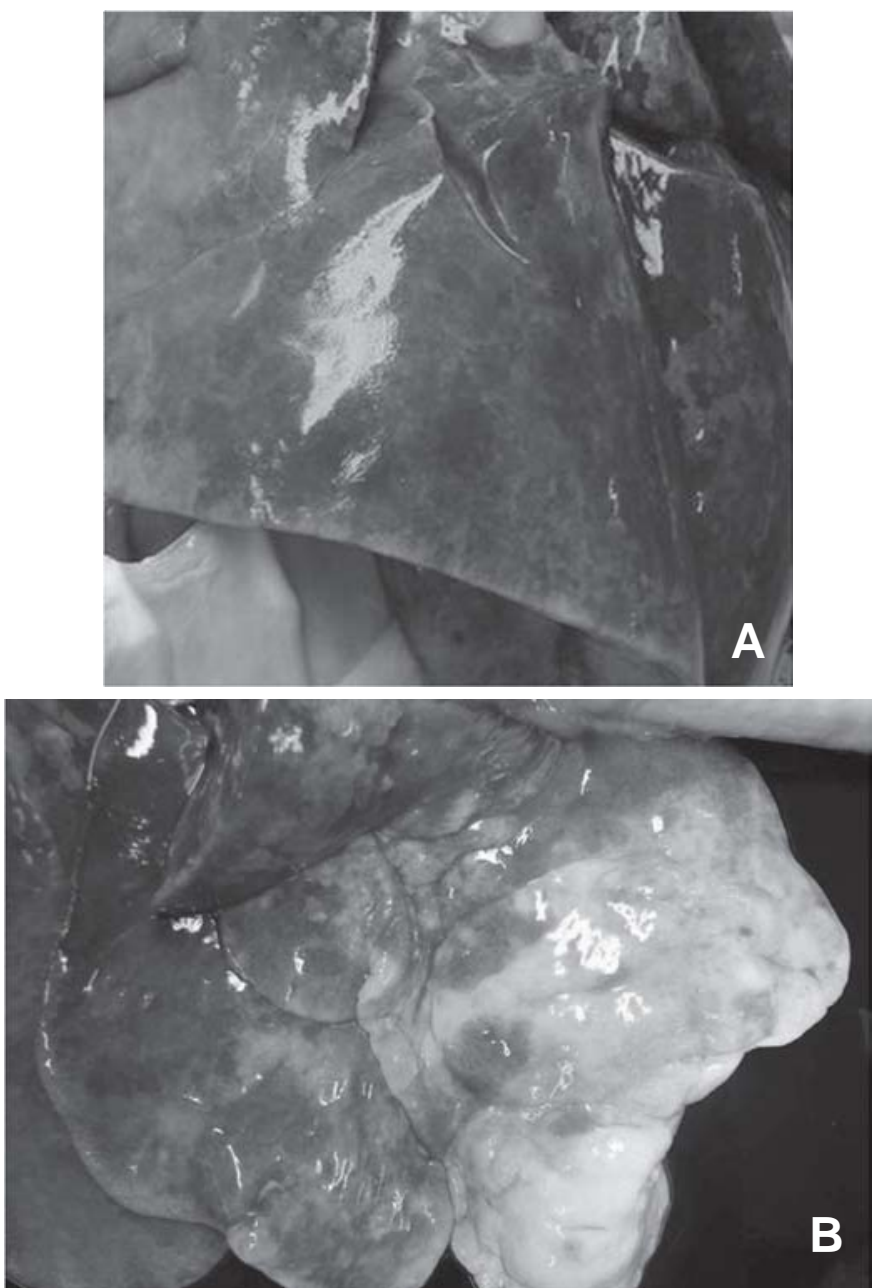

Fig.5. (A) Áreas de petéquias hemorrágicas e necrose, distribuídas em caráter irregular pelo pulmão. (B) Pontos de enfisema em lobos pulmonares caudo-dorsais associados a áreas de efusão pleural.
A silhueta cardíaca mostrou-se aumentada em todos os animais avaliados. Em projeção látero-lateral, o coração apresentou-se sempre em posição verticalizada, chamando particular atenção para os aumentos cardíacos de átrio e ventrículo esquerdos (Fig.2a), que proporcionaram um deslocamento dorsal da região da carina e brônquio principal esquerdo. Em projeção dorsoventral, o coração apresentou-se em forma de "D" invertido (Fig.2b).

A cúpula diafragmática apresentou modificações da morfologia de inserção dos pilares diafragmáticos, observados radiograficamente como um deslocamento caudal que também aumentou a profundidade dos ângulos costofrênicos (Fig.1c, 2a). A flacidez da cúpula diafragmática em sua porção central foi evidenciada através de uma saculação e conseqüente protrusão da mesma para o interior da cavidade torácica localizando-se em mediastino caudal, dificultando sua a visualização (Fig.2b).

A avaliação da estrutura esofágica evidenciou áreas

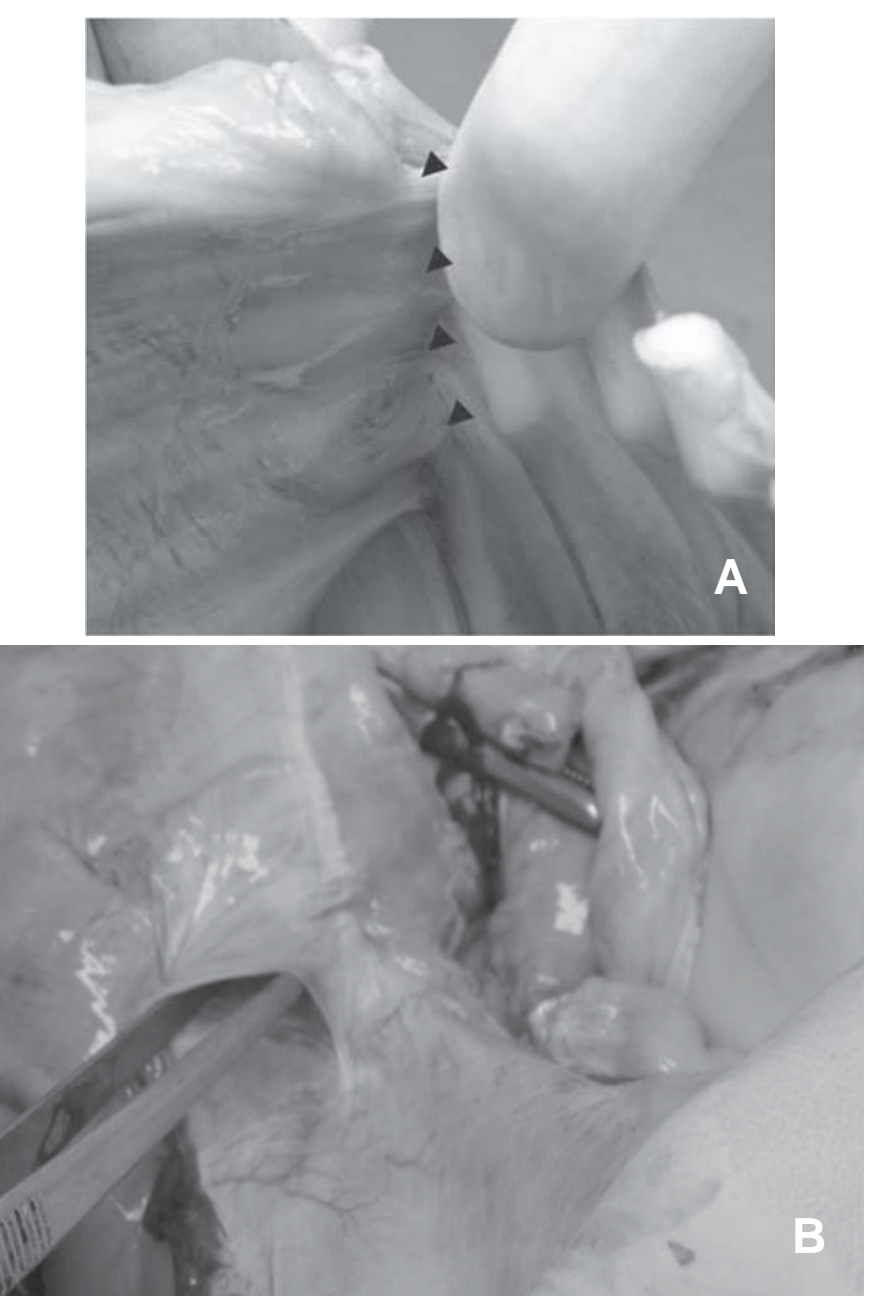

Fig.6. (A) Imagem de necropsia evidenciando espessamento dos pilares diafragmáticos (setas azuis). (B) Observa-se substituição fibrosa progressiva do músculo diafragmático e a presença de uma saculação formada para o interior da cavidade torácica e formação de um divertículo para o interior da cavidade torácica. 
de aerofagia em esôfago cervical, observada em oito dos 10 animais estudados (Fig.3a). A estrutura do esôfago mostrou-se intensamente alterada em todos os animais avaliados, com presença de distensão do lúmen esofágico em região torácica dorsal, com imagem compatível com megaesôfago torácico, vista através da avaliação radiográfica simples (Fig.3b) e em radiografias contrastadas (Fig.3c). Em diversos momentos o estômago foi visualizado repleto por gás e em protrusão para o interior da cavidade torácica, apresentando imagem sugestiva de hérnia hiatal (Fig.4). A hepatomegalia foi um achado observado em pelo menos sete dos 10 animais avaliados.

Os achados de necropsia dos animais que foram a óbito revelaram áreas de petéquias hemorrágicas e necrose, distribuídas de forma irregular pelo pulmão (Fig.5a), associados a pontos de enfisema em lobos pulmonares caudodorsais e efusão pleural (Fig.5b). A cúpula diafragmática apresentou pilares diafragmáticos espessados e a característica translúcida da musculatura intercostal (Fig.6a). A região central apresentou atrofia da musculatura associada à perda e substituição progressiva por tecido fibroso formando uma saculação para o interior da cavidade torácica e com a formação de um divertículo para o interior da cavidade torácica (Fig.6b).

\section{DISCUSSÃO}

A distrofia muscular humana e a estudada em cães da raça Golden Retriever apresentam características semeIhantes com relação a alguns achados clínicos. A disfunção respiratória observada nos cães atendidos, onde se destacaram a pneumonia com áreas de consolidação pulmonar marcante é compatível com as observações feitas por Bergman et al. (2002) e Nguyen et al. (2002), quando estudaram os mecanismos de paralisação e atrofia da musculatura responsável em auxiliar a respiração, facilitando o acúmulo de líquidos pulmonares, levando à evolução para quadros pneumônicos.

A metaplasia observada em nossos achados foi causada pela substituição do tecido muscular por tecido fibroso com conseqüente degeneração das fibras musculares, tornam as mesmas flácidas e incapazes de promover um peristaltismo adequado, levam a um quadro de megaesôfago torácico severo, corroborando com os achados de Valentine et al. (1990) e Hulland (2003), quando em seus estudos com em cães distróficos. Tais alterações foram fortemente observadas na estrutura do músculo diafragma, demonstrando aparência translúcida, perda de elasticidade e incapacidade resistir ao aumento da pressão intra-abdominal, o que pode levar a ruptura diafragmática em sua região central ou formação de um divertículo (saculação), como o observado nos animais desse estudo. Valentine et al. (1990), em estudo morfológico do diafragma de cães com seis meses de idade, também verificou um severo aumento de fibras necróticas com presença de extensa fibrose endomisial e perimisial, ocorrendo infiltrado de tecido adiposo.

Estrutura do miocárdio embora mais acometida em cães adultos, como nos estudos realizados por Valentine et al. (1986, 1990), observou-se aumento cardíaco com áreas de hipertrofia mais evidentes em câmaras cardíacas direita, com o coração em situação de "D" invertido e discreto aumento em região de aorta e átrio esquerdo.

\section{CONCLUSÃO}

A avaliação radiográfica constituiu-se como um meio diagnóstico auxiliar essencial na identificação de doença cardíaca e respiratória em cães Golden Retriever acometidos pela Distrofia Muscular, capaz de identificar processos pneumônicos primários, permitindo o estabelecimento de terapêutica adequada de tratamento, com prognóstico reservado nos estágios mais avançados desta alteração.

\section{REFERÊNCIAS}

Bergman R.L., Inzana K.D., Monroe W.E., Shell L.G., Liu L.A., Engvall E. \& Shelton G.D. 2002. Dystrophin-deficient muscular dystrophy in a Labrador Retriever. J. Am. Anim. Hospital Assoc. 38:255-261.

Cooper B.J., Winand N.J., Stedman H., Valentine B.A., Holffman E.P., Kunnel L.M., Scott M.O., Fischbeck K.H., Kornegay J.N. \& Avery R.J. 1988. The homologue of Duchenne locus is defective in X-linked muscular dystrophy of dog. Nature 334:154-156.

Hulland J.H. 2003. Muscle and tendon, p.253-267. In: Jubb K.V.F., Kennedy P.C. \& Palmer N. (Ed.), Patology of Domestic Animals. 4th ed. Academic Press, San Diego.

Kornegay J.N., Sharp N.J., Bartlett R.J., Van Camp S.D., Burt C.T., Hung W.Y., Kwock L. \& Roses A.D. 1990. Golden Retriever Muscular Dystrophy: monitoring for success. Adv. Exp. Med. Biology 280:267-272.

Myiazato L.G. 2005. Distrofia Muscular no Golden Retriever. Dissertação de Mestrado em Patologia Animal, Faculdade de Ciências Agrárias e Veterinárias, Universidade Estadual Paulista, Jaboticabal. 82p.

Nguyen F., Cherel Y., Guigand L., Goubault-Leroux I. \& Wyers M. 2002. Muscle lesion associated with dystrophin deficiency in neonatal Golden Retriever puppies. J. Comp. Pathol. 126:100-108.

Ronnblon A. \& Danielsson A. 2004. Hereditary muscular disease and symptoms from the gastrintestinal tract. Scand. J. Gastroenterol. 1:14.

Strober J.B. 2006. Therapeutics in Duchenne Muscular Dystrophy. Am. Soc. Exp. Neurotherap. 3(2):225-234.

Valentine B.A., Cooper B.J., Cummings J.F. \& Delahunta A. 1986. Progressive muscular dystrophy in a Golden Retriever dog: light microscopy and ultraestructural features at 4 and 8 months. Acta Neuropathol., Berlin, 71:301-310.

Valentine B.A., Cooper B.J., Cummings J.F. \& Delahunta A. 1990. Canine X-linked muscular dystrophy: morphologic lesions. J. Neurol. Sci. 97:123.

Valentine B.A. \& Cooper B.J. 1991. Canine X-linked muscular dystrophy: selective envolvement of muscle in neonatal dogs. Neuromuscular Disorders 1:31-38.

Valentine B.A., Winand N.J., Pradhan D., Moise N.S., Delahunta A., Kornegay J.N. \& Cooper B.J. 1992. Canine X-linked dystrophy as an animal model of Duchenne Muscular Dystrophy: A review. J. Med. Genetics 42:352-356. 